\title{
Characterization of Mycobacteria in HIV/AIDS Patients of Nepal
}

\author{
'Dhungana GP, ${ }^{2}$ Ghimire P, ${ }^{3}$ Sharma S, ${ }^{4}$ Rijal BP \\ ${ }^{1}$ Department of Microbiology, Siddhanath Science Campus, Mahendranagar, Kanchanpur. ${ }^{2}$ Central department of Microbiology, TU, \\ Kirtipur, Kathmandu, ${ }^{3}$ Department of Medicine, Tribhuvan University, TU, Kathmandu, ${ }^{4}$ Department of Microbiology, TUTH, Kathmandu, \\ Nepal.
}

\begin{abstract}
Besides Mycobacterium tuberculosis, a number of other Mycobacterium species are also occasional human pathogens. Tuberculosis due to Mycobacterium avium complex (MAC) and Mycobacterium kansasii is particularly prevalent in AIDS patients as compared to the normal population. A crosssectional study was carried out during January 2004 to August 2005 in 100 HIV-infected persons visiting Tribhuvan University, Teaching Hospital, and about a dozen of HIV/AIDS care centers of Kathmandu with the objectives to characterize the different mycobacterial species in HIV/AIDS patients. Three sputum specimens from each person were used to investigate tuberculosis by ZiehlNeelsen staining, culture and identification tests. Among the 100 HIV-infected cases, 66 (66\%) were males and 34 (34\%) were females. Sixty percent of the cases were in the age group of 21-30 years. Mycobacteria were detected in 23 (23\%) HIV cases of which 15 (65.2\%) were in the age group of 21-30 years ; 17(74\%) were males and $6(26 \%)$ were females. Among 23 co-infected cases, 22 were culture positive for mycobacteria. Among these, the predominant one was Mycobacterium avium complex (MAC), 9 (41\%), followed by M. tuberculosis, 6 (27\%), M. kansasii, 4 (18\%), M. fortuitum, 2 (10\%) and M. chelonae 1 (4\%). Significant relationship was established between smoking/alcoholism and the subsequent development of tuberculosis $(\chi 2=7.24, \mathrm{p}<0.05$ for smoking habit and $\chi 2=4.39, \mathrm{p}<0.05$ for alcoholisn). Fourteen (61\%) co-infected cases presented with weight loss and cough whereas diarrhea was presented only by those patients with atypical mycobacterial co-infection, which was as high as $5(56 \%)$ in patients with MAC co-infection. This study demonstrated the predominance of atypical mycobacteria, mainly MAC, in HIV/AIDS cases and most of them were from sputum smear-negative cases.
\end{abstract}

Key words: Human Immunodeficiency Virus, mycobacteria, tuberculosis

\author{
Correspondence: \\ Mr. Govinda Prasad Dhungana \\ Department of Microbiology \\ Siddhanath Science Campus, Mahendranagar, \\ Kanchanpur. \\ Email: dhunganagovinda7826@yahoo.com
}


Dhungana et al. Characterization of Mycobacteria in HIV/AIDS Patients of Nepal

\section{INTRODUCTION}

Untreated Human Immunodeficiency Virus (HIV) infection leads to progressive immunodeficiency and increased susceptibility to infection. As HIV infection progresses, $C D+T$-lymphocytes decline in number and function. Thus, the immune system becomes less able to prevent the growth and multiplication of microorganisms and ultimately the patients suffers from a group of several diseases termed acquired immunodeficiency syndrome (AIDS). ${ }^{1}$ Among different types of opportunistic infections in people living with HIV/AIDS (PLWHA), the diseases caused by Mycobacterium species are very common, causing significant mortality and morbidity. Thus on the basis of pathogenesis and the severity of the disease caused by them, mycobacteria are divided into two broad groups- $M$. tuberculosis (the causative agent of tuberculosis) and non-tuberculous mycobacteria (NTM). The other terms for NTM are mycobacteria other than tuberculosis (MOTT), environmental mycobacteria or atypical mycobacteria. ${ }^{2}$ In fact, tuberculosis kills more people than any other single infectious disease and its fatality rate is extremely high in parternership with HIV/AIDS, causing one third of the deaths of PLWHA. ${ }^{3}$ Nepal is considered one of the countries with a high prevalence of tuberculosis and the emergence of HIV has further complicated the matter causing serious public health problems. Additionally, the incidence of MOTT as pathogens in an immuno-compromised host is increasing. A large number of MOTT are frequently found in the environment and can easily colonize in HIV/AIDS patients and cause disease, particularly in the advanced AIDS stage. ${ }^{2}$ Among the MOTT, the most common opportunistic pathogen isolated from HIV/ AIDS patients is the Mycobacterium avium complex (MAC) which is associated with disseminated disease. ${ }^{4}$ Persons with HIV infection and CD4 ${ }^{+} \mathrm{T}$ cell counts less than $100 / \mathrm{mm}^{3}$ have a probability of $10 \%$ to $20 \%$ per year of developing MAC disease or bacteremia. ${ }^{5,6}$

M. kansasii is the second most common MOTT to affect patients with HIV/AIDS accounting for $2.9 \%$ of the MOTT that cause disseminated infection with HIV/AIDS. ${ }^{2,7,8}$ Other MOTT that are less frequently encountered include $M$. fortuitum, M. chelona, M.simiae, etc. ${ }^{2}$

The symptoms and clinical manifestation of MOTT infection mimic tuberculosis. The patients often present non-specific symptoms of cough, fever, night sweat, weight loss, diarrhea and abdominal pain. Any organ can be involved leading to pericarditis, lymph node infection, bone infection, central nervous infection, etc. The introduction of highly-active antiretroviral therapy has resulted in a remarkable decline in MAC infection and death among people living with AIDS. ${ }^{2,4}$

Tuberculosis in HIV/AIDS patients is still a major public health problem in Nepal. It was observed that during
1991-2000 out of 473 AIDS cases, 312 (66\%) cases were co-infected with TB. $^{9}$ Studies conducted at United Mission Hospital, Tansen, Palpa, showed that TB prevalence in HIV patients sharply increased from $10.8 \%$ in 2002 to $39.5 \%$ in $2004 .^{10,11}$

As HIV is fueling mycobacterial disease, it can be expected that the distribution of different species of mycobacteria among HIV patients changes over time. Studies from the developed world identified the different non-tuberculous mycobacterial immune reconstitution syndrome in HIV patients and observed that a specific dose and combination of therapies can reduce the incidence of disseminated MAC infection even in patients with lower CD4 cell count in this era of effective antiretroviral therapy. 12, 4 However, until now in Nepal, both the tuberculous and nontuberculous mycobacteria are treated in the same way, probably due to the lack of policies regarding the laboratory investigations and treatment of nontuberculous mycobacteria. It has been felt that specific studies on tuberculosis in HIV/AIDS patients with the characterization of mycobacteria in such patients to the species level are lacking in the present context of Nepal. So, this study has been conducted with the objectives of measuring the species prevalence of mycobacteria in HIV-infected persons and to characterize the HIV/ AIDS as well as TB/HIV co-infected Nepalese visiting a tertiary care university hospital and different HIV/AIDS care centers of Kathmandu valley which have patient coverage throughout the country.

\section{MATERIAL AND METHODS}

A cross-sectional study was conducted by the Central Department of Microbiology, Tribhuvan University, Kirtipur, in collaboration with Tribhuvan University Teaching Hospital (TUTH), Maharajgunj, during January 2004 to August 2005. Altogether, 100 HIV-infected persons were identified at out-patients/indoor sections of TUTH and at different HIV/AIDS care centers/clinics - Nava Kiran Plus, Sparsha Nepal, Karuna Bhavan, Sneha Samaj, Maiti Nepal, Nepal Plus, Vision plus, SACTS-VCT, Nepal Youth, Aastha Positive Group and Blue Diamond Society. Soon after the approval of Nepal Health Research Council, patients were selected randomly, informed consent was taken and questionnaires were filled followed by three sputum specimen collection from each person. Diagnosis of tuberculosis was done by conventional methods such as direct microscopy of AFB stained smear, AFB culture and identification tests. In direct microscopy, three sputum specimens, i.e. first spot specimen, early morning specimen and second spot specimen (as per WHO recommendation), were collected. ${ }^{3}$ They were stained by Ziehl-Nelsen staining technique and then reporting was done according to the WHO/IUATLD grading system. ${ }^{13}$ In the cultural 
Dhungana et al. Characterization of Mycobacteria in HIV/AIDS Patients of Nepal

technique, one early morning specimen was subjected to modified Petroff's method for decontamination and then a 400 micro-liter deposit was inoculated into a $3 \%$ Ogawa medium followed by incubation at $37^{\circ} \mathrm{C}$ for 8 weeks. In the identification tests, the observation of growth rate and pigmentation, Niacin test, nitrate reeducates test and catalase test were performed according to the WHO manual. ${ }^{13}$

The data obtained from the questionnaires and biochemical test results were entered into statistical Package for social Sciences (SPSS) version 11.5. Mean, median, range and other relevant statistical tools were applied.

\section{RESULT}

One hundred HIV-infected persons were enrolled in this study of which $66(66 \%)$ were male and $34(34 \%)$ were female. Sixty percent were in the age group 2130 years, the mean and median age being 30 years and 28.2 years respectively (Table 1$)$.

The overall prevalence of mycobacterial infection was $23 \%$. Twenty-two species of mycobacteria were isolated of which 4 were smear-positive. The remaining one case was diagnosed only by smear positive result, i.e. it was culture negative (Table 2).

The predominant species was Mycobacterium avium complex (9 out of 22 isolates), followed by M.tuberculosis (6 isolates) as shown in Table 3.

Patients with tuberculosis were very young, the mean age 25.5 years, ranging from 22 years to 27 years whereas patients colonized with Mycobacterium avium complex were comparatively older, the mean age being 35.5 years, ranging from 24 to 54 years. Patients

Table 1. Socio-demographic Characteristics of Studied Cases

\begin{tabular}{lcc}
\hline \multicolumn{1}{c}{ Variable } & number * \\
\hline Age group (Yrs) & $11-20$ & $2(2)$ \\
& $21-30$ & $60(60)$ \\
& $31-40$ & $31(31)$ \\
& $41-50$ & $5(5)$ \\
& $51-60$ & $2(2)$ \\
\hline Sex & Total & $100(100)$ \\
& Male & $66(66)$ \\
\hline Habit & Female & $34(34)$ \\
& Smoker & $41(41)$ \\
\hline
\end{tabular}

* Figures in brackets indicate percent.
Table 2. Modalities of Diagnosis

\begin{tabular}{lccc}
\hline AFB Culture & \multicolumn{2}{c}{ AFB staining } & Total \\
& Positive & Negative & \\
\hline Culture positive & 4 & 18 & 22 \\
Culture negative & 1 & 77 & 78 \\
Total & 5 & 95 & 100 \\
\hline
\end{tabular}

Table 3. Identification of Mycobacterium Species in HIV Cases

\begin{tabular}{lcc}
\hline Species & $\begin{array}{c}\text { Number of } \\
\text { Isolates }\end{array}$ & Percent \\
\hline M. avium Complex & 9 & 41 \\
M. tuberculosis & 6 & 27 \\
$M$. kansasii & 4 & 18 \\
M. fortuitum & 2 & 10 \\
$M$. chelonae & 1 & 4 \\
\hline Total & $22 *$ & 100 \\
\hline
\end{tabular}

* One species couldn't be identified because it was culture negative.

colonized with other mycobacteria were mostly middleaged. Patients infected with M.tuberculosis were all males where as both sexes were affected by MOTT (male : female $=10: 6)$. Among 23 co-infected persons, $15(65.2 \%)$ were smokers and $12(52.2 \%)$ were alcoholics whereas among 77 non co-infected persons, only $26(33.8 \%)$ were smokers and $22(28.6 \%)$ were alcoholics. Thus, significant relationship was established between smoking/alcoholic habit and the subsequent development of tuberculosis $(\chi 2=7.24, p<0.05$ for smoking habit and $\chi 2=4.39, p<0.05$ for alcoholic habit). The major clinical manifestation was found to be cough and weight loss (61\% each). Patients coinfected with $M$. tuberculosis revealed persistence cough as the major clinical manifestation (67\%); none of them presented with diarrhea whereas diarrhea was found to be the major clinical manifestation of patients colonized with MAC (56\%) and other MOTT. Other symptoms like weigh loss, fever, chest pain, etc, were more or less similarly distributed in all mycobacteria (Table 4).

MAC, M. avium Complex; MTB, M. tuberculosis; MK, M. kansasii; MF, M. fortuitum; MCh, M. chelonae, Unidet $=$ Unidentified 
Dhungana et al. Characterization of Mycobacteria in HIV/AIDS Patients of Nepal

Table 4. Sociodemographic Characteristics and Clinical Features of Co-infected Cases.

\begin{tabular}{|c|c|c|c|c|c|c|c|c|}
\hline \multicolumn{2}{|c|}{ Variables } & \multirow{2}{*}{$\frac{\text { MAC }}{3(33.3)}$} & \multirow{2}{*}{$\frac{\text { MTB }}{6(100)}$} & \multirow{2}{*}{$\frac{\text { MK }}{3(75)}$} & \multirow{2}{*}{$\frac{\text { MF }}{2(100)}$} & \multirow{2}{*}{$\begin{array}{c}\text { M.Ch } \\
0\end{array}$} & \multirow{2}{*}{$\frac{\text { Unidet }}{1}$} & \multirow{2}{*}{$\frac{\text { Total }}{15(65.2)}$} \\
\hline Age group & $21-30$ & & & & & & & \\
\hline & $31-40$ & $4(4.44)$ & 0 & $1(25)$ & 0 & $1(100)$ & 0 & $6(26)$ \\
\hline & $41-50$ & $1(11.11)$ & 0 & 0 & 0 & 0 & 0 & $1(4.3)$ \\
\hline & $51-60$ & $1(11.11)$ & 0 & 0 & 0 & 0 & 0 & $1(4.3)$ \\
\hline & Mean age(Yrs) & 35.5 & 25.5 & 28 & 25.5 & 35.5 & 25.5 & 30.3 \\
\hline & Age range & $24-54$ & $22-27$ & $23-39$ & $24-27$ & - & - & $22-54$ \\
\hline \multirow[t]{2}{*}{ Sex } & Male & $6(67)$ & $6(100)$ & $3(75)$ & $1(50)$ & 0 & $1(100)$ & 17 (73.9) \\
\hline & Female & $3(33)$ & 0 & $1(25)$ & $1(50)$ & $1(100)$ & 0 & $6(26.1)$ \\
\hline \multirow[t]{2}{*}{ Smoking } & Yes & $5(56)$ & $4(67)$ & $2(50)$ & $2(100)$ & $1(100)$ & $1(100)$ & $15(65.2)$ \\
\hline & No & $4(44)$ & $2(33)$ & $2(50)$ & 0 & 0 & 0 & $8(34.8)$ \\
\hline \multirow[t]{2}{*}{ Alcohol } & Yes & $4(44)$ & $4(67)$ & $2(50)$ & $1(50)$ & 0 & $1(100)$ & $12(52)$ \\
\hline & No & $5(56)$ & $2(33)$ & $2(50)$ & $1(50)$ & $1(100)$ & 0 & $11(48)$ \\
\hline \multirow[t]{5}{*}{ Cl. features } & Weight loss & $5(56)$ & $2(33)$ & $3(75)$ & $2(100)$ & $1(100)$ & $1(100)$ & $14(61)$ \\
\hline & Fever & $5(56)$ & $2(33)$ & $2(50)$ & $2(100)$ & 0 & $1(100)$ & $12(52)$ \\
\hline & Chest pain & $3(33)$ & $1(16)$ & $2(50)$ & $1(50)$ & 0 & $1(100)$ & $8(36)$ \\
\hline & Cough & $5(56)$ & $4(67)$ & $2(50)$ & $1(50)$ & $1(100)$ & $1(100)$ & $14(61)$ \\
\hline & Night sweat & $4(44)$ & $1(16)$ & $3(75)$ & $2(100)$ & 0 & $1(100)$ & 11 (48) \\
\hline
\end{tabular}

* Figures in brackets indicate percentage.

\section{DISCUSSION}

One of the most important and new findings of this study was the high prevalence of atypical mycobacteria, mainly Mycobacterium avium complex $(41 \%$ of the 22 isolates) and M. kansasii (18\% of total isolates) in HIV/ AIDS patients of Nepal, suggesting that these patients were in the advanced stage of immunodeficiency. A study conducted by the international MAC Study Group concluded that low CD4 count was associated with an increased disseminated MAC infection. ${ }^{14}$ Simillarly, Mycobacterium avium complex was found to be the predominant species isolated from HIVinfected patients of Colombia affecting $4.5 \%$ of the HIV-infected patients, followed by Mycobacterium tuberculosis (1.4\%). ${ }^{15}$ Another similar study conducted in HIV/AIDS patients at Muniz Hospital in Buenos Aires documented that $5.8 \%$ of the total mycobacterial isolates were MAC. ${ }^{16}$ Moreover, the same study documented $19 \%$ mycobacterial disease among the HIV patients there, which is close to our finding of $23 \%$ mycobacterial disease among HIV patients. This prevalence rate suggests the increasing trend of TB among HIV patients of Nepal. During the late 1990s, TB/HIV co-infection was $6 \%$ in Kathmandu ${ }^{17}$ whereas the prevalence of TB in HIV patients visiting United
Mission Hospital, Tansen, Palpa, sharply increased from $10.8 \%$ in 2002 to to $39.5 \%$ in $2004 .{ }^{10}, 11$ Moreover, our findings of TB status among HIV patients was consistent with the WHO/UNAIDS report stating that one-third of HIV/ AIDS patients were co-infected with TB. ${ }^{18}$ However, our study showed the surprisingly higher prevalence of MAC. This may be due to the following reasons: patients possibly in the advanced stage of HIV infection with very low CD4 count, lack of appropriate treatment directed against MAC infection Nepal, patients having undergone DOTS therapy (directed against $M$. tuberculosis but not against MAC) and chance of patients' selection (i.e. selection bias). Furthermore, we would like to stress that this finding cannot be generalized to reflect the HIV community of Nepal because the cases were selected only from HIV/ STI clinics/ care homes and the TUTH OPD/hospital but not from the community.

In our study, we observed that only $21.7 \%$ of the total TB (including mycobacterial disease) cases were smearpositive. This value is lower than the value obtained at Muniz Hospital (Buenos Airers) where $59 \%$ of the pulmonary cases were smear-positive ${ }^{16}$. Another study conducted in the general population (House hold survey) of Nepal also showed that the distribution of smear-positive TB is lower in comparison to smear- 
negative TB $(41.4 \%$ smear-positive TB versus $58.6 \%$ smear negative TB $).{ }^{19}$ As our study is conducted in HIV patients $21.7 \%$ smear positive may be considered usual in the context of Nepal. Furthermore, in a document published by WHO, it was stated that sputum smear negative TB cases constituted the major proportion of HIV patients, particularly in late stage of HIV infection. ${ }^{3}$

The high incidence of mycobacterial colonization in the male of age group 21-30 years could be due to two reasons. Firstly, the majority of the HIV infected people fall in this age group and sex; secondly, the males of this age group had relatively higher exposure to the outside environment. This finding is similar to the findings of similar studies in different settings .10, 20

The clinical manifestation of the patients demonstrated the association of $M$. tuberculosis mainly with primary lungs infection whereas of MAC with dessiminated infection. Other similar studies have also documented the disseminated syndromes due to MAC. ${ }^{12}$ Significant relationship between smoking/alcoholic and development of disease suggests that these habits may be the risk factors of mycobacterial disease.

\section{CONCLUSION}

This study demonstrated the higher prevalence of MAC $(41 \%)$ than of $M$. tuberculosis (27\%) in HIV/ AIDS patients of Nepal. The majority of the co-infected persons were sputum smear-negative cases, suggesting the value of culture in screening tuberculosis in HIV patients. This study highlights the necessity of adopting new policies regarding the surveillance and treatment of MAC infection in HIV patients. Weight loss and cough were found to be the major clinical features in co-infected patients and these parameters would be helpful for surveillance purpose. Besides these, habits like smoking and alcohol addiction were found to be the major risk factors for co-infection.

\section{ACKNOWLEDGEMENT}

The authors would like to acknowledge Tribhuvan University Teaching Hospital for giving permission to use the laboratory facilities and Nepal Health Research Council (NHRC) for providing the research grant. We express our sincere thanks to different HIV/STI clinics of the valley for their help during specimen collection.

\section{REFERENCES}

1. World Health Organization. TB/HIV clinical Manual. 2nd ed. Geneva: WHO; 2004. p. 27-9.

2. Grange JM. Tuberculosis. In: collier L, Balows A, Sussman M, editoes. Topley and Wilson's Microbiology and Microbial infections. 9th ed. Philadelphia: Edward Arnold; 1997. p.391-437.

3. World Health Organization. Tuberculosis Handbook. Geneva: WHO; 1998. p.9-72.

4. Lange CG, Woolley IJ, Brodt RH. Disseminated Mycobacterium avium - intracellulare complex (MAC) infection in the era of effective antiretroviral therapy: Is prophylaxis still indicated? Drugs 2004; 64(7):67992.

5. Chaisson RE, Moore RD, Richman DD, Keruly J, Creagh T. Incidence and Natural History of Mycobacterium avium complex in patients with advanced HIV disease treated with Zidovudine. The Zidovudine study epidemiology study group. Am Rev Respir Dis 1992;146:285-9.

6. Nightingle SD, Byrd LT, Southern PM, Jockusch JD, Wynne BA. Incidence of Mycobacterium avium intracellulare bacteremia in HIV positive patients J infect Dis 1992;165:1082-5.

7. Bamberger DM, Driks MR. Mycobacterium kansasii infection in the acquired immunodeficiency syndrome (AIDS) in kansas city. Cli Inf Dis 1994;18:395-400.
8. Forbes BA, Sahm DF, weissfeld AS. Bailey and Scott's Diagnostic Microbiology. 11th ed. Baltinore: Mosby Inc; 2002. p.538-69.

9. Subedi BK. HIV-TB co-infection in Nepal. J Inst Med 2003;25:19-21.

10. Ghimire P, Dhungana JR, Bam DS, Rijal BP. Tuberculosis and Human immunodeficiency virus Coinfection in United Mission Hospital-Tansen. SAARC J Tuber, HIV/ AIDS and Lung Dis 2004;1(1):32-8.

11. Luitel BR, Lamgade A, Busal L, Napit I. Trends of HIV infection in United Mission Hospital, Tansen - A retrospective Glimpse. J Nepal Med Assoc 2005;44(16):63.

12. Phillips P, Bonner S, Gataric C, Bai T, Wilcox P, Hogg $\mathrm{R}$, et al. Non tuberculous mycobacterial immune reconstitution syndrome in HIV infected patients: Spectrum of disease and long term follow - up. Cli Inf Dis 2005;41(10):1483-97.

13. World Health Organization. Laboratory Services in Tuberculosis Control. Geneva: WHO; 1998. p.58-75.

14. Fordham RC, Arbeit RD, Tosteson AN, Ristola MA, Barber TW, Waddel R, et al. The international Epidemiology of disseminated Mycobacterum avium complex in AIDS. AIDS 1996;10(9):1025-32.

15. Murcia-Aranguren MI, Gómez-Marin JE, Alvarado 
FS, Bustillo JG, de Mendivelson E, Gómez B, et al. Frequency of tuberculous and non-tuberculous mycobacteria in HIV infected patients from Bogota, Colombia. BMC Infect Dis. 2001;1:21. Epub 2001 Nov 8.

16. Di Lonardo M, Isola NC, Ambroggi M, Rybko A, Poggi S. Mycobacteria in HIV infected persons in Buenos Aires. Tuber and Lung Dis 1995;76(3):185-9.

17. Sherchand JB, Bam DS, Sherchand S. Human Immunodefieicncy Virus (HIV) infection in Tuberculosis patients of Nepal. Jour Nepal Assoc for Med Lab Sci 2001;4(4):1-5.
18. Sharma SK, Mohan A, Kadhiravum T. HIV/TB Coinfection: Epidemiology, diagnosis and management. Ind Jour of Med Resear 2005;121:550-7.

19. Martin A, Preston C, Byanjankar L, Bam DS, Pande SB, Baral SC, et al. Estimated number of new TB cases in Patan, A city in Nepal. Jour of Health Org and Mgmt 2007; 21(6):546-53.

20. Muniyandi M, Rajeswari R, Balasubramanian R. Tuberculosis Control Programme - Is it pro poor? SAARC J Tuber, HIV/ AIDS and Lung Dis 2004;1(1):149. 